Published in final edited form as:

Addict Behav. 2017 May ; 68: 35-38. doi:10.1016/j.addbeh.2017.01.014.

\title{
Prevalence and correlates of fentanyl-contaminated heroin exposure among young adults who use prescription opioids non-medically
}

\author{
Alexandria Macmadu ${ }^{a, b}$, Jennifer J. Carroll ${ }^{c, d}$, Scott E. Hadland ${ }^{e}$, Traci C. Green ${ }^{f, g, h}$, and \\ Brandon D. L. Marshall ${ }^{\text {h }}$ \\ aThe Center for Prisoner Health and Human Rights, The Miriam Hospital, 164 Summit Ave, \\ Providence, RI, 02906, USA \\ bDepartment of Health Services, Policy and Practice, Brown University School of Public Health, \\ 121 South Main Street, Box G-S-121-7, Providence, RI, 02912, USA \\ 'The Division of Infectious Diseases, The Miriam Hospital, 164 Summit Ave, Providence, RI, \\ 02906, USA \\ dDepartment of Medicine, The Warren Alpert School of Medicine of Brown University, 222 \\ Richmond Street, Providence, RI, 02903, USA \\ eDepartment of Pediatrics, Boston University School of Medicine, 88 East Newton Street, Boston, \\ MA, 02118, USA \\ fDepartment of Emergency Medicine, Boston University School of Medicine, 771 Albany Street, \\ Boston, MA, 02118, USA \\ 9Department of Emergency Medicine, The Warren Alpert School of Medicine of Brown University, \\ 222 Richmond Street, Providence, RI, 02903, USA \\ hDepartment of Epidemiology, Brown University School of Public Health, 121 South Main Street, \\ Box G-S-121-2, Providence, RI, 02912, USA
}

\section{Abstract}

Background-The rate of overdose deaths caused by fentanyl-contaminated heroin (FCH) use is increasing rapidly in the United States. We examined risk factors for exposure to $\mathrm{FCH}$ and experiences with FCH use among young adult non-medical prescription opioids (NMPO) users.

\footnotetext{
Send correspondence to: Brandon D. L. Marshall, PhD, Manning Assistant Professor of Epidemiology, Department of Epidemiology, Brown University School of Public Health, 121 South Main Street, Box G-S-121-2, Providence, RI 02912, USA, brandon_marshall@brown.edu.

Publisher's Disclaimer: This is a PDF file of an unedited manuscript that has been accepted for publication. As a service to our customers we are providing this early version of the manuscript. The manuscript will undergo copyediting, typesetting, and review of the resulting proof before it is published in its final citable form. Please note that during the production process errors may be discovered which could affect the content, and all legal disclaimers that apply to the journal pertain.

Contributors: All authors made significant contributions to the study's design and conduct. AM was the lead writer and conducted study analyses. BDLM and AM developed the research question and drafted the analysis plan. BDLM, JJC, SEH, and TCG critiqued the analysis plan, assisted with the interpretation of findings, and contributed to multiple versions of this brief. All authors contributed to and have approved the final manuscript for publication.

Conflict of interest: No conflict declared.
} 
Methods-We analyzed data from the Rhode Island Young Adult Prescription Drug Study (RAPiDS), which enrolled young adults aged 18 to 29 reporting prior 30 day NMPO use between January 2015 and February 2016. Participants completed questionnaires ascertaining drug use patterns and risk behaviors, including FCH exposure. Logistic regression was used to assess factors associated with known or suspected FCH exposure.

Results-Of 199 participants, the median age was 25 (IQR: 22, 27), 130 (65.3\%) were male, and $122(61.3 \%)$ were of white, non-Hispanic race/ethnicity. In total, 22 (11\%) reported known or suspected FCH exposure in the prior six months. Several drug use patterns and risk behaviors were associated with $\mathrm{FCH}$ exposure, including: regular heroin and cocaine use; diverted pharmaceutical fentanyl use in the prior six months; NMPO use to avoid withdrawal symptoms; longer duration of NMPO use; injection drug use; and prior overdose (all $p<0.001$ ). Among participants who reported $3 \mathrm{FCH}$ exposure, 59\% were unaware that their heroin was contaminated with fentanyl prior to last use, 59\% reported that FCH provides a better high, and all recognized that fentanyl increases overdose risk.

Conclusions-Exposure to fentanyl-contaminated heroin is an emerging trend among young adult NMPO users in Rhode Island. Overdose prevention programs addressing FCH use are urgently needed.

\section{Keywords}

fentanyl; heroin; non-medical prescription opioid use; young adults

\section{INTRODUCTION}

Opioid use disorder and opioid overdose are escalating epidemics in the United States. From 2000 to 2014, the rate of opioid-involved overdose deaths increased by 200\% (Rudd, Aleshire, Zibbell, \& Gladden, 2016). During this period, non-medical prescription opioid (NMPO) use was a driver of overdose-related mortality (Calcaterra, Glanz, \& Binswanger, 2013). However, more recent national data suggest shifting trends, with heroin-involved overdose deaths increasing more than three-fold between 2010 and 2014 (Compton, Jones, \& Baldwin, 2016).

Across the US, and particularly in New England, rates of fatal overdose have been exacerbated by fentanyl-contaminated heroin $(\mathrm{FCH})$ and other forms of illicit fentanyl use (Centers for Disease Control and Prevention, 2015). From 2013 to 2014, overdose death rates involving synthetic opioids (including fentanyl) increased 80\% (Rudd et al., 2016). In 2015, half of all overdose deaths in Rhode Island involved fentanyl and fentanyl analogsan increase from 37\% in 2014 and less than 5\% in the years prior to 2014 (Rhode Island Governor's Overdose Prevention and Intervention Task Force, 2015). Eighty-six fentanyl deaths occurred in Maine in 2015, up from only 2 in 2013 (Sorg, Greenwald, \& Wren, 2016), and fentanyl was attributed to five times as many deaths as heroin in 2015 in New Hampshire (2016). Given this emerging crisis, there is an urgent need to identify persons who may be at risk for fentanyl-related overdose and to understand users' experiences with $\mathrm{FCH}$. 
The purpose of this study was to identify correlates of self-reported use of heroin that is known or suspected to have been adulterated with fentanyl (hereafter referred to as FCH exposure) and to examine users' experiences with FCH. To the authors' knowledge, this study is among the first to explore correlates of $\mathrm{FCH}$ exposure and examine firsthand experiences with and attitudes toward FCH among young adults in the US.

\section{MATERIALS AND METHODS}

\subsection{Study design and sample}

The data analyzed here were collected as a part of a pilot study that assessed the patterns and determinants of NMPO use among young adults. From January 2015 to February 2016, the Rhode Island Young Adult Prescription Drug Study (RAPiDS) recruited 200 Rhode Island residents aged 18-29 who endorsed prior-30-day NMPO use. Participants were compensated $\$ 25$ for their time. This study was approved by the Institutional Review Board (IRB) at Brown University.

Participants were recruited through a combination of targeted canvassing, snowball sampling, and internet-based recruitment from online classifieds, drug information websites, and social media. Throughout the recruitment period, paper recruitment flyers were placed in and distributed from Rhode Island-based substance use treatment centers, recovery support centers, urgent care centers, and service agencies (e.g., syringe exchange programs); street canvassing was also utilized in major Rhode Island cities.

Snowball sampling methods were utilized in the first recruitment phase. During this phase, each participant was asked to refer up to two eligible peers. For referrals that were redeemed, the referring participant received one $\$ 5$ gift card via mail or in person. Referred participants were also invited to refer their peers to the study, and so forth, until several recruitment chains were produced.

Internet-based recruitment methods were utilized in the second recruitment phase. Participants were recruited from online classified sites (e.g., Craigslist), drug information sites (e.g., Bluelight.org), and social media sites (e.g., Facebook). Search engine-based banner advertising was also used to target Rhode Island-based individuals searching relevant terms (e.g., opioid, pills).

Participants were interviewed individually by trained personnel in private settings. Computer-assisted personal interviewing (CAPI) was used to collect socio-demographic data and drug use patterns; computer-assisted self-interviewing (CASI) was used to collect particularly sensitive or stigmatizing information (e.g., injection drug use).

\subsection{Measures}

2.2.1 Non-medical fentanyl use —-All participants were asked whether they had ever engaged in non-medical fentanyl use, defined as use "without a doctor's orders or not as a doctor directed". Participants who endorsed lifetime non-medical fentanyl use were asked which formulations of fentanyl they had used in the prior six months. Response options included "skin patch", "pills", "nasal spray", "lozenge/lollipop", "a film that dissolves under 
your tongue", and "fentanyl-laced heroin". Participants who endorsed this last option were considered as having known or suspected FCH exposure in the prior six months; an affirmative answer to any other response option was defined as any diverted fentanyl use in the prior six months. Those who endorsed FCH use were asked whether-at their most recent use of $\mathrm{FCH}$ - they were aware of fentanyl contamination prior to use. We also assessed frequency of FCH use in the prior six months (response options were "never", "once or a couple of times", "about once a month", "at least every week", and "every day"), and we solicited experiences with and attitudes toward FCH. Covariates of interest were socio-demographic characteristics, drug use patterns and risk behaviors, and clinical factors.

2.2.2 Socio-demographic characteristics-We assessed age, male sex at birth, race/ ethnicity (categorized as white, non- Hispanic vs. other), educational attainment beyond high school, as well as lifetime history of detainment in a jail/prison and homelessness.

2.2.3 Risk behaviors and drug use patterns-Drug use patterns assessed included regular use of heroin, NMPO use, cocaine use, injection drug use, and non-medical benzodiazepine use, which we defined as weekly or greater in the prior six months. Nonmedical prescription drug use was defined as use "without a doctor's orders or not as a doctor directed," and to improve measurement validity, the images and brand names of several prescription opioids (e.g., Vicodin, OxyContin) and benzodiazepines (e.g., Xanax, Valium) were included in the assessment. Participants reported their duration of NMPO use (in years), as well as whether they primarily use prescription opioids to avoid withdrawal symptoms. We also assessed lifetime history of witnessing and experiencing an overdose.

2.2.4 Clinical factors-We examined history of any mental health diagnosis, self-reported positive hepatitis $\mathrm{C}$ virus (HCV) status among those who had ever tested for $\mathrm{HCV}$, and whether participants had ever been prescribed an opioid.

\subsection{Statistical analyses}

Given the low prevalence of FCH exposure in the sample, bivariable logistic regression was used to assess factors associated with the outcome. Descriptive statistics were generated to characterize experiences with and attitudes toward $\mathrm{FCH}$. One participant was excluded due to missing data. Statistical analyses were conducted using SAS version 9.4. All $p$-values are two-sided.

\section{RESULTS}

Among the 199 participants who were included in these analyses, the majority was male (65.3\%), and the median age was 25 (interquartile range [IQR]: 22-27). While the majority was white/non-Hispanic (61.3\%), collectively, a substantial fraction of the sample was black (16.6\%), multi-racial (15.1\%), or ethnically Latino/a (14.1\%). The prevalence of FCH exposure in the prior six months was $11.1 \%(\mathrm{n}=22)$. Along with age, white race/ethnicity, and lifetime homelessness, FCH exposure was significantly associated with several drug use patterns and risk behaviors, including: regular heroin and cocaine use; regular injection drug use, diverted pharmaceutical fentanyl use in the prior six months; regular non-medical benzodiazepine use, NMPO use primarily to avoid withdrawal symptoms; and longer 
duration of NMPO use (all $p<0.05$, see Table 1). The majority of participants reporting FCH exposure had ever experienced an overdose (63.6\%), compared to only $22.0 \%$ of nonexposed participants (unadjusted odds ratio $=6.2,95 \% \mathrm{CI}: 2.4-15.8$ ). All but one participant reporting FCH exposure had ever witnessed an overdose. Self-reported positive HCV status was also associated with FCH exposure in the prior six months (see Table 1).

Among those who reported FCH exposure in the prior six months, when asked about their most recent suspected exposure to $\mathrm{FCH}, 13$ (59\%) reported being unaware that the heroin was contaminated with fentanyl prior to use (i.e., were told that their drugs contained fentanyl after use or suspected exposure based on their reaction to the drug); $8(36 \%)$ reported being aware that the heroin was contaminated with fentanyl prior to use, and $1(5 \%)$ was unsure. Three (14\%) reported daily use of FCH and $8(36 \%)$ reported using FCH "at least every week."

Attitudes toward FCH varied. Among participants who reported FCH exposure, 14 (64\%) reported that they were confident that they could distinguish FCH from heroin that is not contaminated with fentanyl; 8 (36\%) reported that they were not confident or unsure. Among those who were unaware that their heroin was contaminated with fentanyl prior to use, $6(46 \%)$ agreed or strongly agreed that $\mathrm{FCH}$ provides a better high than heroin that is not contaminated with fentanyl; among those who were aware, 7 (88\%) agreed or strongly agreed that $\mathrm{FCH}$ provides a better high $(p=0.174)$. Altogether, 59\% $(\mathrm{n}=13)$ reported that FCH provides a better high. All participants either agreed $(n=3,14 \%)$ or strongly agreed $(\mathrm{n}=19,86 \%)$ that fentanyl contamination increases overdose risk.

\section{DISCUSSION}

The present study is among the first to explore correlates of FCH use and report firsthand experiences with and attitudes toward FCH among young adults in the US. Among young adult NMPO users, those who endorsed FCH use typically reported greater and more extensive drug use experiences, as evidenced by more frequent and longer durations of drug use, injection drug use, greater endorsement of risk behaviors, and worse health outcomes (e.g., self-reported $\mathrm{HCV}$ positive status). The majority reported being unaware that their heroin was contaminated with fentanyl at their most recent use, and those who were aware were more likely to report that $\mathrm{FCH}$ provides a better high. All participants acknowledged that fentanyl contamination increases overdose risk.

Correlates of FCH use have not been previously explored among young adults in the US; however, our findings are consistent with research examining factors associated with nonfatal overdose among young people who use opioids non-medically. Several factors identified in previous studies as correlates of non-fatal overdose were significantly associated with FCH use in the present study, including white racial background (Sherman, Cheng, \& Kral, 2007), longer duration of drug use (Schrager et al., 2014; Sherman et al., 2007), regular non-medical benzodiazepine use and heroin use (Silva, Schrager, Kecojevic, \& Lankenau, 2013), and injection drug use (Mitra, Wood, Nguyen, Kerr, \& DeBeck, 2015; Richer, Bertrand, Vandermeerschen, \& Roy, 2013; Schrager et al., 2014; Sherman et al., 2007; Silva et al., 2013; Werb, Kerr, Lai, Montaner, \& Wood, 2008). Therefore, individuals 
with FCH exposure appear to be at highest risk for overdose. To mitigate fentanyl-related overdose risk, tailored prevention and harm reduction that engage young adults with more extensive NMPO and other drug use experience are needed. For example, a recent analysis of the same study sample found that fewer than half of study participants were aware of the Rhode Island Good Samaritan Law, which provides legal protections to individuals who witness an overdose (Evans, Hadland, Clark, Green, \& Marshall, 2016). Campaigns to encourage young adults to call 911 in the event that they witness an overdose are needed. Programs to increase naloxone distribution and training to young adults who use prescription opioids non-medically are also needed (Marshall, Green, Yedinak, \& Hadland, 2016).

To our knowledge, firsthand experiences with and attitudes toward FCH have not been explored in the US in recent decades. One study from 1983 found that, among 18 selfidentified users of fentanyl in California, many were not aware of the fentanyl contamination prior to use, and respondents perceived fentanyl contamination to increase overdose risk (LaBarbera \& Wolfe, 1983). These results are consisted with present findings. Of major public health concern is our finding that the majority of participants who reported $\mathrm{FCH}$ exposure had used it without knowing that their heroin was contaminated with fentanyl. To inform public health efforts that address $\mathrm{FCH}$, future research should examine whether fentanyl contamination can be reliably identified prior to consumption (using rapid tests, for example) (Amlani et al., 2015).

There are some limitations to this study. First, as our analyses were derived from a pilot study, the sample size is relatively small, which may have resulted in type I or II error. Second, our study likely underestimates the proportion of young adult NMPO users who have experience with FCH because some participants may have used FCH without their knowledge; the experiences of these individuals are not reflected here. Third, our study relies on self-report, as we did not collect biological confirmatory data. Additional research using toxicological analysis is needed to determine the prevalence of and risk factors for fentanyl exposure among young adults who use drugs. Finally, reported confidence in ability to identify fentanyl-contaminated heroin may not be reliable, as interpretations of this item may have varied (i.e., identify visually prior to use vs. identify sensation following use); for this reason, the proportion of young adult NMPO users who have experience with FCH might also be overestimated. Given these limitations, the findings from this study should be interpreted as preliminary and warrant additional research. Nonetheless, our findings underscore the urgent need for effective overdose prevention in this vulnerable population.

\section{Acknowledgments}

We give special thanks to the study participants for their contribution to the research, as well as current and past RAPiDS researchers and staff. We would specifically like to thank Jesse Yedinak and Beth Elston for their research and administrative assistance.

Role of the funding source: The RAPiDS project is supported by the US National Institute on Drug Abuse [grant number R03-DA037770]. Dr. Marshall is supported by a Henry Merrit Wriston Fellowship from Brown University. Dr. Hadland is supported by the Leadership Education in Adolescent Health Training Program T71 MC00009 $(\mathrm{MCH} / \mathrm{HRSA})$ and a National Research Service Award from the Eunice Kennedy Shriver National Institute of Child Health and Human Development [grant number 1T32 HD075727]. Dr. Green is supported by the Agency for Healthcare Research and Quality [grant number R18 HS024021] and the Centers for Disease Control and Prevention [grant number R21 CE002165]. Dr. Carroll is supported by a training grant from the National Institute on Drug Abuse to the Division of Infectious Diseases at The Miriam Hospital [grant number T32 DA013911]. The 
sponsors had no role in the study design; the collection, analysis and interpretation of data; the writing of the report; and in the decision to submit the article for publication.

\section{REFERENCES}

Amlani A, McKee G, Khamis N, Raghukumar G, Tsang E, Buxton JA. Why the FUSS (Fentanyl Urine Screen Study)? A cross-sectional survey to characterize an emerging threat to people who use drugs in British Columbia, Canada. Harm reduction journal. 2015; 12(1):1.

Calcaterra S, Glanz J, Binswanger IA. National trends in pharmaceutical opioid related overdose deaths compared to other substance related overdose deaths: 1999-2009. Drug and alcohol dependence. 2013; 131(3):263-270. [PubMed: 23294765]

Centers for Disease Control and Prevention. CDC Health Advisory: Increases in Fentanyl Drug Confiscations and Fentanyl-related Overdose Fatalities. HAN Health Advisory. 2015 Oct 26. 2015.

Compton WM, Jones CM, Baldwin GT. Relationship between Nonmedical Prescription-Opioid Use and Heroin Use. New England Journal of Medicine. 2016; 374(2):154-163. [PubMed: 26760086]

Evans TI, Hadland SE, Clark MA, Green TC, Marshall BD. Factors associated with knowledge of a Good Samaritan Law among young adults who use prescription opioids non-medically. Harm Reduct J. 2016; 13(1):24. [PubMed: 27455957]

LaBarbera M, Wolfe T. Characteristics, attitudes and implications of fentanyl use based on reports from self-identified fentanyl users. Journal of psychoactive drugs. 1983; 15(4):293-301. [PubMed: 6655528]

Marshall BD, Green TC, Yedinak JL, Hadland SE. Harm reduction for young people who use prescription opioids extra-medically: Obstacles and opportunities. International Journal on Drug Policy. 2016

Mitra G, Wood E, Nguyen P, Kerr T, DeBeck K. Drug use patterns predict risk of non-fatal overdose among street-involved youth in a Canadian setting. Drug and alcohol dependence. 2015; 153:135139. [PubMed: 26096535]

New Hampshire Office of the Chief Medical Examiner. New Hampshire Drug Overdose Deaths by Year. 2016.

Rhode Island Governor's Overdose Prevention and Intervention Task Force. Rhode Island's Strategic Plan on Addiction and Overdose: Four Strategies to Alter the Course of an Epidemic. 2015. Retrieved from Rhode Island Department of Health: https://static1.squarespace.com/static/ 55d208f6e4b04baeaff02618/t/5644d6f6e4b07080d3aba45f/1447352054184/Task+Force+Strategic + Plan.pdf

Richer I, Bertrand K, Vandermeerschen J, Roy É. A prospective cohort study of non-fatal accidental overdose among street youth: The link with suicidal ideation. Drug and alcohol review. 2013; 32(4):398-404. [PubMed: 23130603]

Rudd RA, Aleshire N, Zibbell JE, Gladden RM. Increases in drug and opioid overdose deaths-United States, 2000-2014. MMWR: Morbidity and mortality weekly report. 2016; 64(50-51):1378-1382. Retrieved from http://www.cdc.gov/mmwr/preview/mmwrhtml/mm6450a3.htm. [PubMed: 26720857]

Schrager SM, Kecojevic A, Silva K, Jackson Bloom J, Iverson E, Lankenau SE. Correlates and consequences of opioid misuse among high-risk young adults. Journal of addiction. 2014; 156954

Sherman SG, Cheng Y, Kral AH. Prevalence and correlates of opiate overdose among young injection drug users in a large US city. Drug and alcohol dependence. 2007; 88(2):182-187. [PubMed: 17110058]

Silva K, Schrager SM, Kecojevic A, Lankenau SE. Factors associated with history of non-fatal overdose among young nonmedical users of prescription drugs. Drug and alcohol dependence. 2013; 128(1):104-110. [PubMed: 22974490]

Sorg MH, Greenwald M, Wren JA. Patterns of Drug-induced Mortality in Maine, 2015 Update. Maine Policy Review. 2016; 25(1):34-46.

Werb D, Kerr T, Lai C, Montaner J, Wood E. Nonfatal overdose among a cohort of street-involved youth. Journal of Adolescent Health. 2008; 42(3):303-306. [PubMed: 18295139] 


\section{Highlights}

- $11 \%$ of sample reported recent exposure to fentanyl-contaminated heroin (FCH)

- $\quad$ FCH users reported greater and more extensive drug use experiences

- Most reported that $\mathrm{FCH}$ provides a better high than heroin

- $\quad$ All acknowledged that FCH use increases overdose risk

- Most were unaware that their heroin was contaminated with fentanyl prior to use 
Macmadu et al.

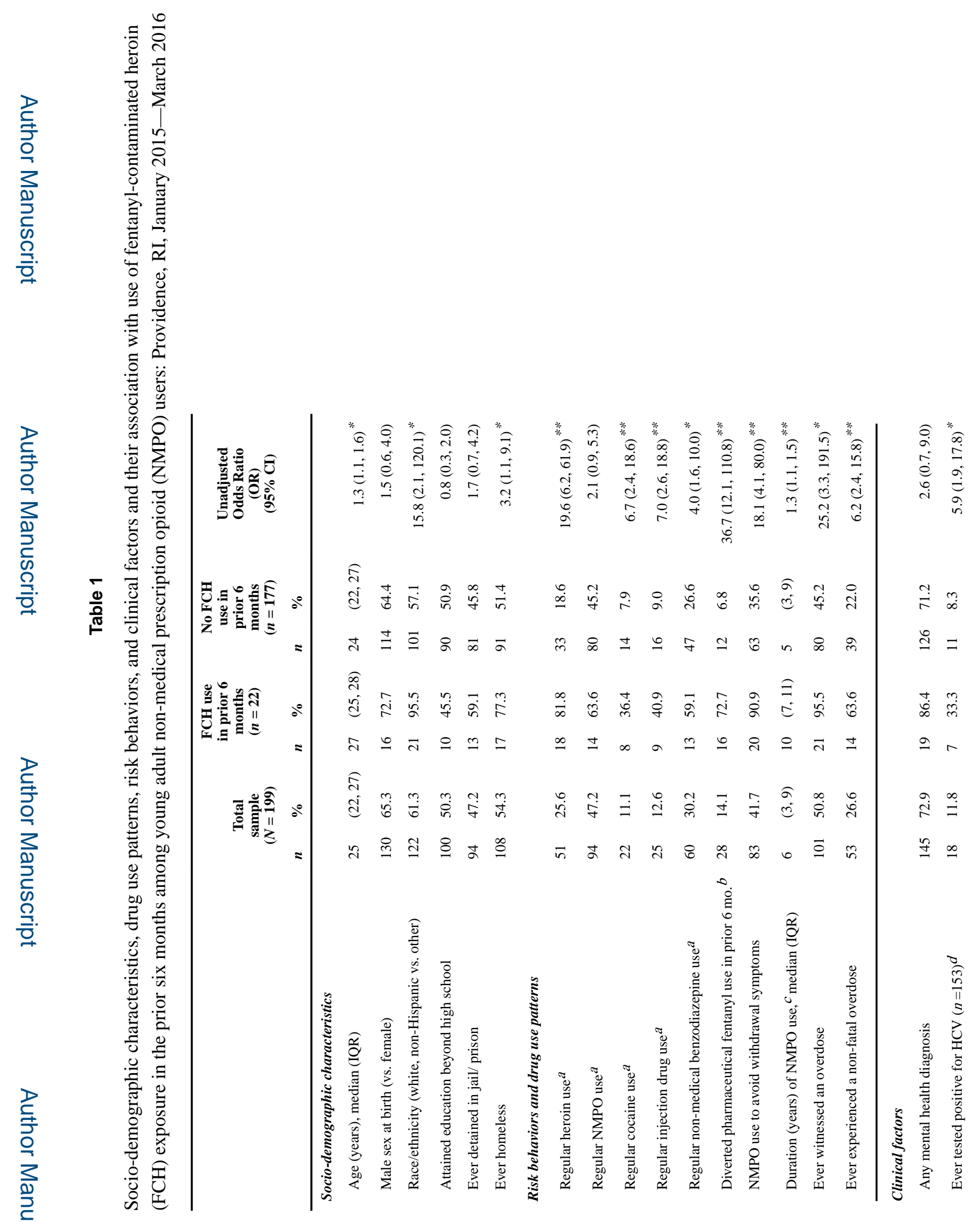




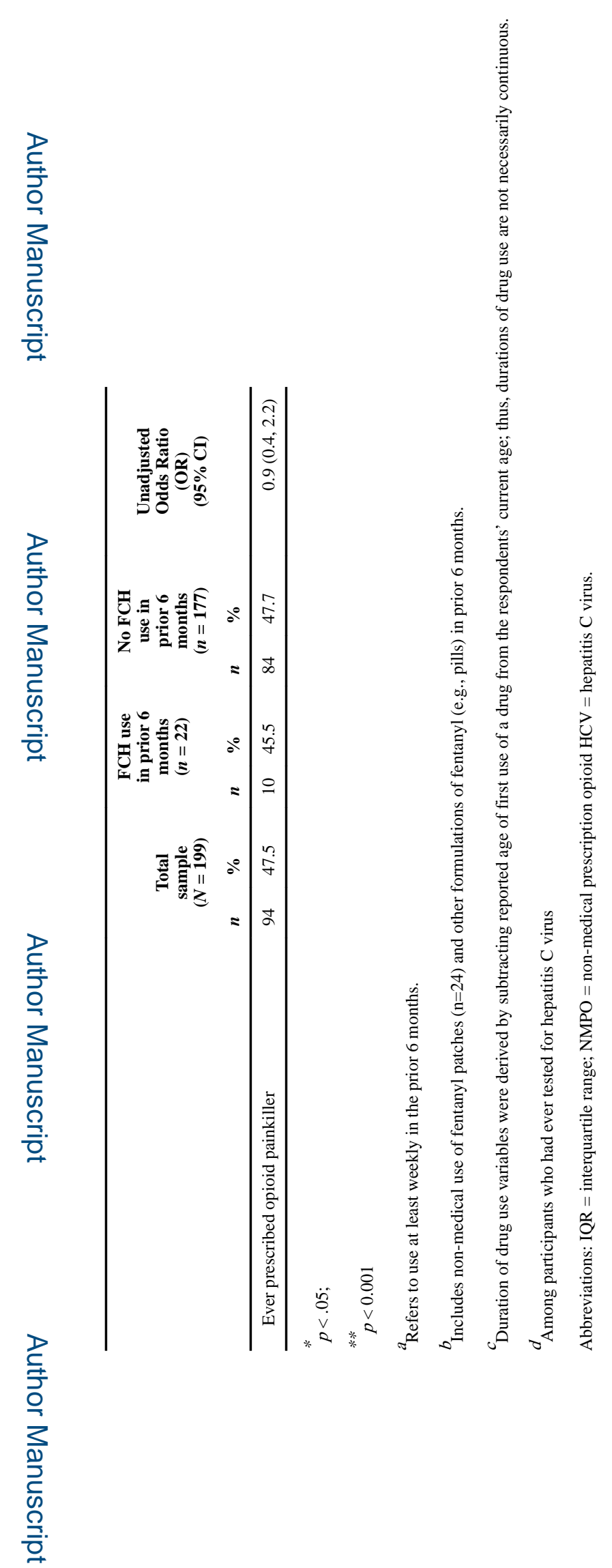

